\title{
Country-Level Factors Dynamics and ABO/Rh Blood Groups Contribution To COVID-19 Mortality
}

\author{
Alfonso Monaco \\ Istituto Nazionale di Fisica Nucleare (INFN), Sezione di Bari \\ Ester Pantaleo \\ Neuroscienze e organi di senso \\ Nicola Amoroso \\ Istituto Nazionale di Fisica Nucleare (INFN), Sezione di Bari

\section{Loredana Bellantuono} \\ Neuroscienze e organi di senso \\ Alessandro Stella ( $\nabla$ alessandro.stella@uniba.it) \\ Università degli Studi di Bari "Aldo Moro" \\ Roberto Bellotti \\ Istituto Nazionale di Fisica Nucleare (INFN), Sezione di Bari
}

\section{Research Article}

Keywords: COVID-19, mortality, prediction, ABO, Rh, blood group

Posted Date: June 14th, 2021

DOl: https://doi.org/10.21203/rs.3.rs-576429/v1

License: (c) (i) This work is licensed under a Creative Commons Attribution 4.0 International License.

Read Full License

Version of Record: A version of this preprint was published at Scientific Reports on December 1st, 2021. See the published version at https://doi.org/10.1038/s41598-021-04162-2. 


\title{
Country-level factors dynamics and $\mathrm{ABO} / \mathrm{Rh}$ blood groups contribution to COVID-19 mortality
}

\author{
Alfonso Monaco ${ }^{1} \mathbb{I}$, Ester Pantaleo $2 \mathbf{q}$, Nicola Amoroso ${ }^{1,3}$, Loredana Bellantuono ${ }^{2}$, \\ Alessandro Stella ${ }^{4^{*}}$, Roberto Bellotti ${ }^{1,5}$ \\ 1 Istituto Nazionale di Fisica Nucleare (INFN), Sezione di Bari, Via A. Orabona 4, 70125 \\ Bari, Italy \\ 2 Dipartimento di Scienze mediche di base, Neuroscienze e organi di senso, Piazza G. \\ Cesare 11, 70124 Bari, Italy \\ 3 Dipartimento di Farmacia - Scienze del Farmaco, Università degli Studi di Bari "Aldo \\ Moro", Via A. Orabona 4, 70125 Bari, Italy \\ 4 Dipartimento di Scienze biomediche e oncologia umana, Università degli Studi di Bari \\ "Aldo Moro", Bari, Italy \\ 5 Dipartimento Interateneo di Fisica "M. Merlin", Università degli Studi di Bari "Aldo \\ Moro", Via G. Amendola 173, 70125 Bari, Italy \\ IThese authors contributed equally to this work. \\ * Corresponding author \\ Email: alessandro.stella@uniba.it
}

\section{ABSTRACT}

Identifying factors related to COVID-19 mortality is important to deploy effective containment measures and to safeguard categories at risk. In the last months, several investigations have tried to ascertain essential features for predicting the COVID-19 mortality tolls depending on country-specific dynamics and population structure. Most studies focused on the initial outbreak of COVID-19 spanning the first half of 2020. Several variables, including obesity, health system indicators such as hospital bed density, and bacillus Calmette-Guerin vaccination have been reported as significantly associated to COVID-19 mortality. Here, we examined in different pandemic stages some of the mentioned associations as well as $\mathrm{ABO}$ and Rh blood group indicators, which have also been previously linked to COVID-19 severity and fatal outcome. Using a machine learning approach, we found that the "B+" blood group frequency is an important factor at all stages of the pandemic.

Keywords: COVID-19, mortality, prediction, ABO, Rh, blood group

\section{INTRODUCTION}

The first information on a cluster of cases of "pneumonia of unknown cause" or "viral pneumonia" was notified to WHO's country offices in the People's Republic of China on December 31st 2019 ${ }^{1}$. Since then, as of April 19th 2021, 141,642,813 global COVID-19 cases have been recorded with more than 3 million deaths ${ }^{2}$. While these numbers would lead to a rough estimate of the case fatality rate (CFR) of around $2.1 \%$ worldwide, wide differences are observed in country-specific death rates. As an example, in the same previously mentioned date of April 19th 2021 the CFR ranged from $9.21 \%$ in Mexico to $0.05 \%$ in Singapore ${ }^{3}$. The reason for this diversity in country specific CFRs has only recently been investigated. Among factors under scrutiny there were comorbidities such as obesity ${ }^{4}$, diabetes $^{4-6}$, high blood pressure ${ }^{7}$, general indicators of the quality of healthcare systems including the number of hospital beds per thousands ${ }^{8}$ or the number of tests per thousands ${ }^{9}$, 
and the age population structure with specific reference to the percentage of residents aged $>70$ where a higher CFR has been generally observed ${ }^{10}$. The large majority of studies published so far, have investigated the contribution of these factors during the first wave of the COVID-19 pandemic. However, two further waves of cases with their load of casualties have been experienced since the release of these first reports. Further, large collaborative studies on COVID-19 cases enrolled during the first wave of the pandemic have identified the first set of genetic loci possibly responsible for the observed wide variation in symptoms severity ${ }^{11-14}$. After the initial outbreak in China, the most severely hit countries were those with a high gross domestic product (GDP) per capita and well-established healthcare systems. In contrast, COVID-19 casualties appeared to be lower in selected world regions such as the Middle East, South Eastern Asia and lower income countries. Several factors can explain the observed differences in the first wave of COVID-19, including under-reporting of cases, limited testing and challenges in the attribution of the cause of death. However, after more than a year of ongoing pandemic, this peculiar geography appears to be changing. In this study, we aimed to investigate the role of country-specific determinants including the genetic structure of local populations in the different waves of the pandemic and to assess how these factors' contribution changed over time. For this purpose we used an approach based on artificial intelligence which is capable of integrating the effects of several factors and their interactions in a multivariate nonlinear model. Using the total deaths per million caused by COVID-19 (TDPM) and indicators such as demographic, economic, health system related and genetic factors, this work provides a quantitative analysis of the SARS-CoV-2 pandemic in several countries through a longitudinal study sampled at three different times: June, September and December 2020. Next, we determined the specific contribution of different features to the pandemic severity. Noticeably, the B+ blood histotype became more important as pandemic progressed while "diabetes prevalence" and "cardiovascular death rate" lost importance. As far as we know, our study is one of the first that combines different kinds of features, including genetic indicators, in a complex forecasting model that exploits the potential of machine learning to study different waves of the COVID-19 pandemic. The reported results could be strategic to better understand its spreading and develop effective measures to reduce its death toll.

\section{METHODS}

\section{Data}

Our aim was to explore whether $\mathrm{ABO}$ and Rhesus blood group frequencies could predict the total deaths per million caused by COVID-19 (TDPM). We explored this relationship at three different time points, June 15 2021, September 15 2021, and December 15 2021, which cover the second half of 2020, i.e. the period corresponding to the end of the first pandemic wave, the beginning of the second outbreak, and the apex of this second stage. To build a prediction model we used a set of 10 indicators of ABO and Rhesus blood group frequencies, or "genetic" features ${ }^{15}$. In addition we included a set of 12 "non-genetic" features downloaded from OWID ${ }^{16}$ and updated to 2020 including demographic, economic, medical, and life style indicators. Table I contains a complete list of the input features used to build a predictive model of the TDPM. To get a wide and varied perspective we used data from 75 worldwide countries listed in Table II (35 European, 21 Asian, 7 African, 6 North American, 4 South American, 2 Oceanian countries).

\section{Data analysis}

For the prediction of the TDPM we used a machine learning approach based on a versatile and non linear machine learning algorithm, the Random Forest (RF) model. The data analysis procedure is summarized in Fig. 1. Given the relatively high mutual correlation of 
TABLE I. List of input features. We used five kinds of features: genetic, demographic, economic, medical, and life style indicators.

\begin{tabular}{|c|c|c|}
\hline Genetic features & Demographic indicators & Medical indicators \\
\hline$O+$ & Population density & Life expectancy at birth \\
$A+$ & Median age of the population & Cardiovascular death rate \\
$B+$ & Population aged 65 or older & Diabetes prevalence \\
$A B+$ & Population aged 70 or older & \\
$O-$ & & \\
$A-$ & Economic indicators & Life style indicators \\
$B-$ & GDP per capita & Percentage of female smokers \\
$A B-$ & Total healthcare expenditure & Percentage of male smokers \\
\cline { 2 - 3 } / non $O$ & Hospital beds per thousand inhabitants & \\
\cline { 2 - 3 }$R-/ R h+$ & & \\
\hline
\end{tabular}

TABLE II. List of input countries.

\begin{tabular}{|c|c|c|}
\hline \multicolumn{3}{|c|}{ Countries } \\
\hline Austria & Belgium & Bosnia and Herzegovina \\
Bulgaria & Cyprus & Croatia \\
Czechia & Denmark & Estonia \\
Finland & France & Germany \\
Greece & Hungary & Iceland \\
Ireland & Italy & Lithuania \\
Luxembourg & Malta & Moldova \\
Montenegro & Netherlands & Norway \\
Poland & Portugal & Romania \\
Russia & Serbia & Slovakia \\
Slovenia & Spain & Sweden \\
Ukraine & United Kingdom & Armenia \\
Bangladesh & Bahrain & China \\
India & Indonesia & Iran \\
Israel & Japan & Lebanon \\
Myanmar & Malaysia & Nepal \\
Philippines & Singapore & Saudi Arabia \\
South Korea & Thailand & Turkey \\
United Arab Emirates & Yemen & Ethiopia \\
Ghana & Kenya & Mauritius \\
Morocco & South Africa & Zimbabwe \\
Canada & Costa Rica & Dominican Republic \\
Jamaica & Mexico & United States \\
Brazil & Chile & Colombia \\
Ecuador & New Zealand & Australia \\
\hline
\end{tabular}

the chosen predictors (see Fig. 2), we fed the RF algorithm with a subset of the whole set of features. We used the Boruta wrapper to select relevant features. We also tested a linear multivariate model for comparison.

\section{Feature selection}

We performed feature selection using a robust and efficient algorithm called Boruta ${ }^{17}$, which is a wrapper method based on the Random Forest algorithm (described in the next section). Briefly, Boruta (Boruta is a god of the forest in the Slavic mythology) exploits the same idea that originates the Random Forest method: it perturbs the system with elements of randomness and computes results from the set of randomized samples, thus decreasing the negative upshot of random instabilities and correlations inherent in a classification or a 


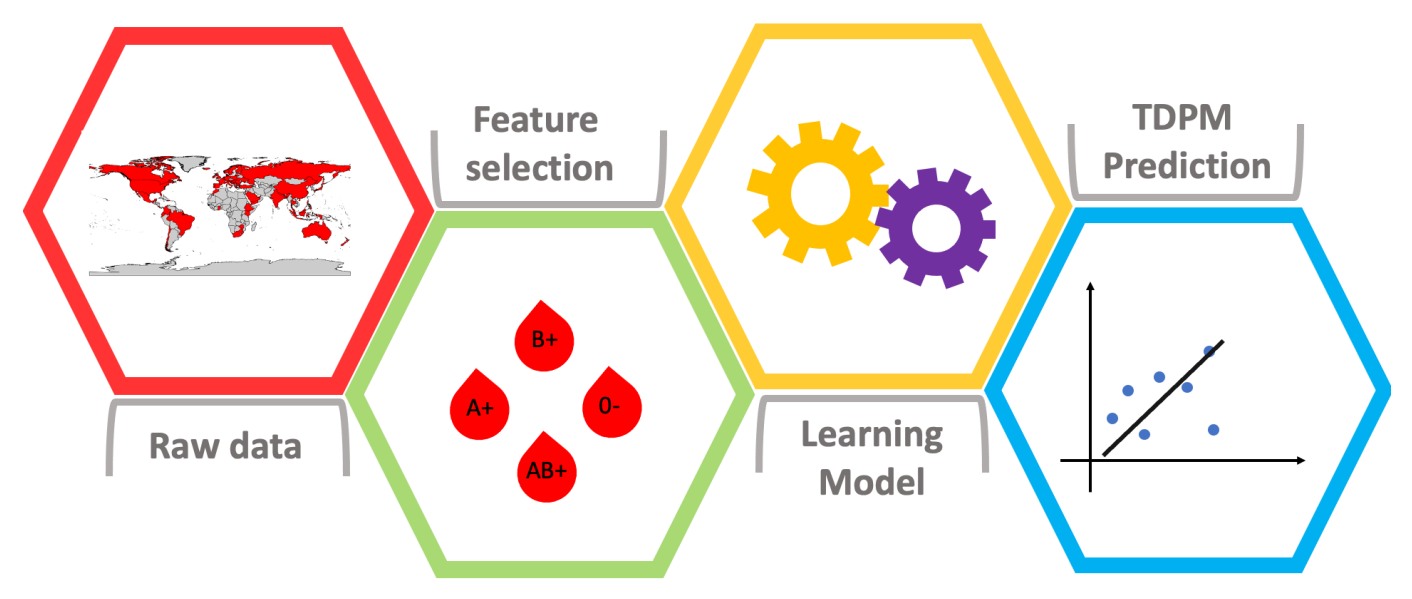

FIG. 1. Flowchart of the proposed methodology. We fed the learning algorithm with selected features to forecast the TDPM.

forecasting algorithm.

During the training phase, Boruta enlarges independent Random Forest trees on different bagging samples building shadow features, or copies of the original features with shuffled values, and compares the importance of the original features with the importance of their random shuffled copies. In other word it uses a permutation procedure to validate the importance assigned to the features by the RF algorithm, increasing the robustness of the methodology: shadow attributes play the role of reference values for deciding which attributes are important. Tentative features have an importance that is so close to their best shadow features that Boruta cannot make a decision with the desired confidence. By design, Boruta selects all features which are relevant to the outcome variable prediction and the selected features yield a minimal forecasting error.

\section{Learning model}

A Random Forest (RF) is composed by an ensemble of classification/regression trees made by means of bootstrapping of the training dataset ${ }^{18}$. Due to a randomization process of the input variables in the training phase, the RF trees have low mutual correlation. In fact, in the building step of the trees, at each node a subset of features is randomly selected. Furthermore, RFs have some characteristics that make them ideal in many machine learning analyses. For instance, they are simple to tune as most of the times they only require modulation of two parameters: the number of trees $n$ and $m$ the number of features sampled to grow each leaf within a tree. Furthermore RF can evaluate the importance of each input feature during the training phase by means of the mean decrease of impurity, averaging over the whole forest of trees ${ }^{18}$. Moreover the RF algorithm is robust against overfitting and through an out-of-bag procedure it provides an unbiased estimate of the generalization error. Because it uses decision trees, the RF algorithm can capture non linear relationships with the input features.

In the present work we implemented a standard configuration in which each forest is composed by $n=500$ trees and $m$ is chosen to give the lowest RMSE. The optimal $m$ is 2 at all time points. 


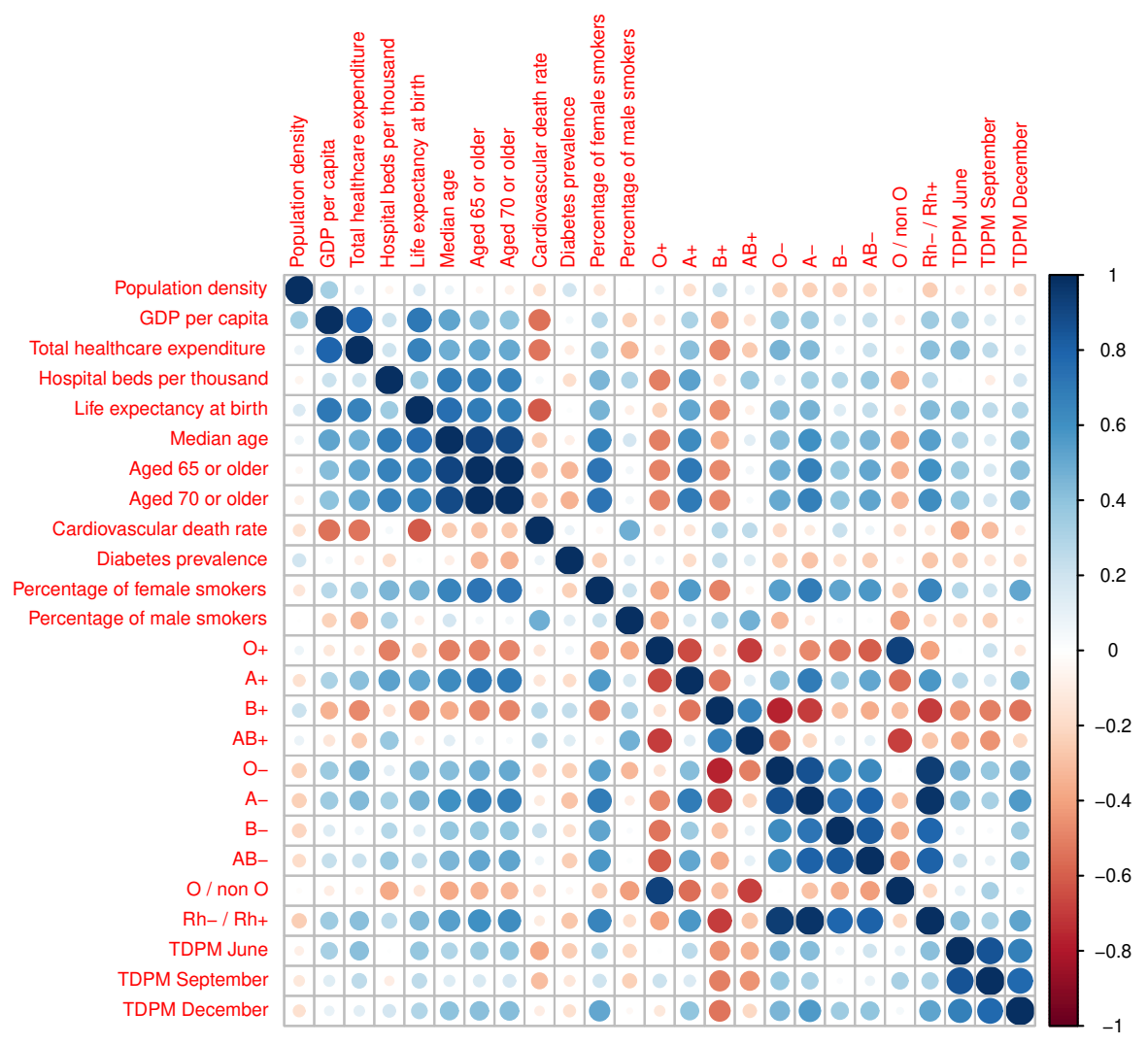

FIG. 2. Correlation matrix of all variables (both dependent and independent). As expected "Median age of the population", "Population aged 65 or older", and "Population aged 70 or older" have mutual correlation close to 1; also the genetic features are highly correlated with each other. The TDPMs at the three different dates are also highly correlated with each other, as expected. Notably, the "B+" predictor has the highest (and negative) linear correlation with the TDPM at all three dates.

\section{Cross Validation and Performance Metrics}

To increase the robustness of our procedure and minimize overfitting issues, we adopted a 5 -fold classification framework. In other words, we divided the initial dataset of 75 nations into 5 random subsets without repetition. We used the union of 5 minus 1 subsets as training set and the remaining set for validation and repeated this procedure five times, which gave us five different training and validation sets, and therefore five models with their respective performances. The average of these five performance values is a reliable indicator of the overall model performance.

We measured performances in terms of coefficient of determination between predicted and actual values $\left(\mathrm{R}^{2}\right)$. In addition we evaluated the root mean square error (RMSE):

$$
R M S E=\sqrt{\frac{1}{N} \sum_{i=i}^{N}\left(A_{t}-F_{t}\right)^{2}}
$$

and the mean absolute error (MAE): 


$$
M A E=\frac{1}{N} \sum_{i=i}^{N}\left|A_{t}-F_{t}\right|
$$

where $A_{t}$ and $F_{t}$ are the actual and the forecast values, respectively. Both data processing and statistical analyses were performed in $\mathrm{R}$ version 3.6.1 ${ }^{19}$.

\section{RESULTS}

We run the Boruta algorithm on the set of 22 input features to predict the TDPM at the three different time points. In all cases, many features assumed similar importance, and tentative and important features were difficult to discern as they were distributed similarly to shadow features (see Supplementary Figure 1 in the Supplementary Information where shadow features are represented in blue, and tentative and important features in yellow and green, respectively). Noticeably, feature "B+", or frequency of blood group "B+", unambiguously stood out as the most important feature, well above other features in June, September and December.

Given the random nature of the Boruta algorithm, to establish which features to select as important among similarly important/tentative features, we run it 500 times with different random seeds and computed the distribution of the Boruta importance measure. At each time point we selected features using a cut-off criterion as follows. First, we excluded features having median below the median of the "Shadow Max" variable, or the shadow variable with the highest median importance (see Fig. 4). Then, among the remaining features, we selected only features whose lower quartile was bigger that the upper quartile of the "Shadow Max" variable (drawn in red in Fig. 4). Using this procedure, we selected frequency of "B+", "Diabetes Prevalence", and "Cardiovascular death rate" in June, "B+" and "AB+" in September ("AB+" was not selected in June because, despite having median importance higher than "Shadow Max"'s median, it did not satisfy the chosen criterion), and six features in December, namely "B+", "O-", "A-", "Rh-/Rh+", "Percentage of female smokers", and "Population density".

To verify the stability of the algorithm with respect to the set of important features selected, we also performed Boruta 100 times (with different random seeds) over the whole dataset and counted the number of times each feature was selected as important by Boruta. Results show that the selected features are stable (see Table III).

We then evaluated the RF regression model with the selected features in terms of $\mathrm{R}^{2}$, RMSE, and MAE with a 5-fold CV procedure. Results are shown in Table IV. Fig. 5 shows the average importance, within the RF model, of each of the selected features with the respective error bars.

Furthermore we used the selected Boruta features as input to a multivariate linear model, to check that the RF improves upon the linear model by adding a level of complexity (compare Tables IV and V), and to compare significant features. Also the linear model finds "B+" important, at a 0.01 significance level, but doesn't find the other features significant except for "Cardiovascular death rate" in June, while the overall multivariate linear model is highly significant with an R-squared ranging between 0.29 and 0.32 (see Table V).

\section{DISCUSSION}

In the first wave of the COVID-19 pandemic, striking differences were reported in the case fatality rate of different countries. While many factors can confound the identification of potential determinants of the death rates caused by COVID-19, several studies have been released in the past months addressing the contribution of different elements to the wide variability in country-specific CFRs. 
TABLE III. Given the random nature of the Boruta algorithm we performed 100 runs of this algorithm on the same dataset with different seeds, then counted how many times each feature was selected by Boruta and reported counts in this table. Column "Type" has value "g" and " $\mathrm{n}$ " for "genetic" and "non genetic" features, respectively.

\begin{tabular}{|c|c|c|c|c|}
\hline Name & Type & $\begin{array}{c}\text { Percente } \\
\text { June }\end{array}$ & $\begin{array}{l}\text { Se of times } \\
\text { September }\end{array}$ & $\begin{array}{l}\text { selected in } \\
\text { December }\end{array}$ \\
\hline$B+$ & $\mathrm{g}$ & 100 & 100 & 100 \\
\hline Diabetes prevalence & $\mathrm{n}$ & 100 & 0 & 0 \\
\hline Cardiovascular death rate & $\mathrm{n}$ & 98 & 20 & 0 \\
\hline$O-$ & $\mathrm{g}$ & 93 & 4 & 100 \\
\hline$A B+$ & $\mathrm{g}$ & 91 & 100 & 0 \\
\hline$R h-/ R h+$ & $\mathrm{g}$ & 42 & 1 & 100 \\
\hline$A-$ & $\mathrm{g}$ & 25 & 49 & 100 \\
\hline Total healthcare expenditure & $\mathrm{n}$ & 4 & 0 & 0 \\
\hline$O+$ & $\mathrm{g}$ & 0 & 2 & 0 \\
\hline Percentage of female smokers & $\mathrm{n}$ & 0 & 0 & 100 \\
\hline Population density & $\mathrm{n}$ & 0 & 0 & 100 \\
\hline$B-$ & $\mathrm{g}$ & 0 & 0 & 75 \\
\hline$A+$ & $\mathrm{g}$ & 0 & 0 & 1 \\
\hline GDP per capita & $\mathrm{n}$ & 0 & 0 & 0 \\
\hline Hospital beds per thousand & $\mathrm{n}$ & 0 & 0 & 0 \\
\hline Life expectancy at birth & $\mathrm{n}$ & 0 & 0 & 0 \\
\hline Median age & $\mathrm{n}$ & 0 & 0 & 0 \\
\hline Aged 65 or older & $\mathrm{n}$ & 0 & 0 & 0 \\
\hline Aged 75 or older & $\mathrm{n}$ & 0 & 0 & 0 \\
\hline Percentage of male smokers & $\mathrm{n}$ & 0 & 0 & 0 \\
\hline$A B-$ & $\mathrm{n}$ & 0 & 0 & 0 \\
\hline$O / \operatorname{non} O$ & $\mathrm{n}$ & 0 & 0 & 0 \\
\hline
\end{tabular}

TABLE IV. Performance measures of the RF regression model at each selected time point, using the selected Boruta features and averaged over 5 runs of cross validation (with the respective standard deviations).

\begin{tabular}{|c|c|c|c|}
\hline Time point & $\mathbf{R}^{2}$ & RMSE & MAE \\
\hline June & $0.47 \pm 0.13$ & $135 \pm 10$ & $85 \pm 11$ \\
September & $0.25 \pm 0.19$ & $192 \pm 37$ & $129 \pm 24$ \\
December & $0.34 \pm 0.04$ & $312 \pm 48$ & $241 \pm 39$ \\
\hline
\end{tabular}

We decided to investigate the number of fatalities due to COVID-19 in relation to the entire population of analyzed countries (i.e., the total deaths/1 million population, TDPM) rather than the more frequently used case fatality ratio (i.e., mortality, CFR). These two parameters are influenced in different ways by multiple variables such as the number and type of diagnostic tests performed in each country or the modalities used to impute deaths to COVID-19. Since the CFR strictly relies on the number of tests performed in each country, and testing has not been homogeneously performed in different countries, we decided to focus on the TDPM parameter.

Features selected as input in our model were a combination of demographic, health and economic indicators, and frequencies of $\mathrm{ABO}$ and $\mathrm{Rh}$ blood groups. We chose $\mathrm{ABO}$ and $\mathrm{Rh}$ since several reports have indicated that both blood groups could influence the probability to progress to severe COVID-19 disease in SARS-CoV-2 infected subjects ${ }^{20-25}$. Further, while additional genetic loci have been identified in genome wide association studies, ABO and $\mathrm{Rh}$ blood groups offer the advantage of having frequencies available for almost all countries in the world. Fig. 3 shows maps of TDPM at the three considered time points and the worldwide distribution of selected blood group frequencies.

In our study we explored the relationship between the total number of deaths per million 

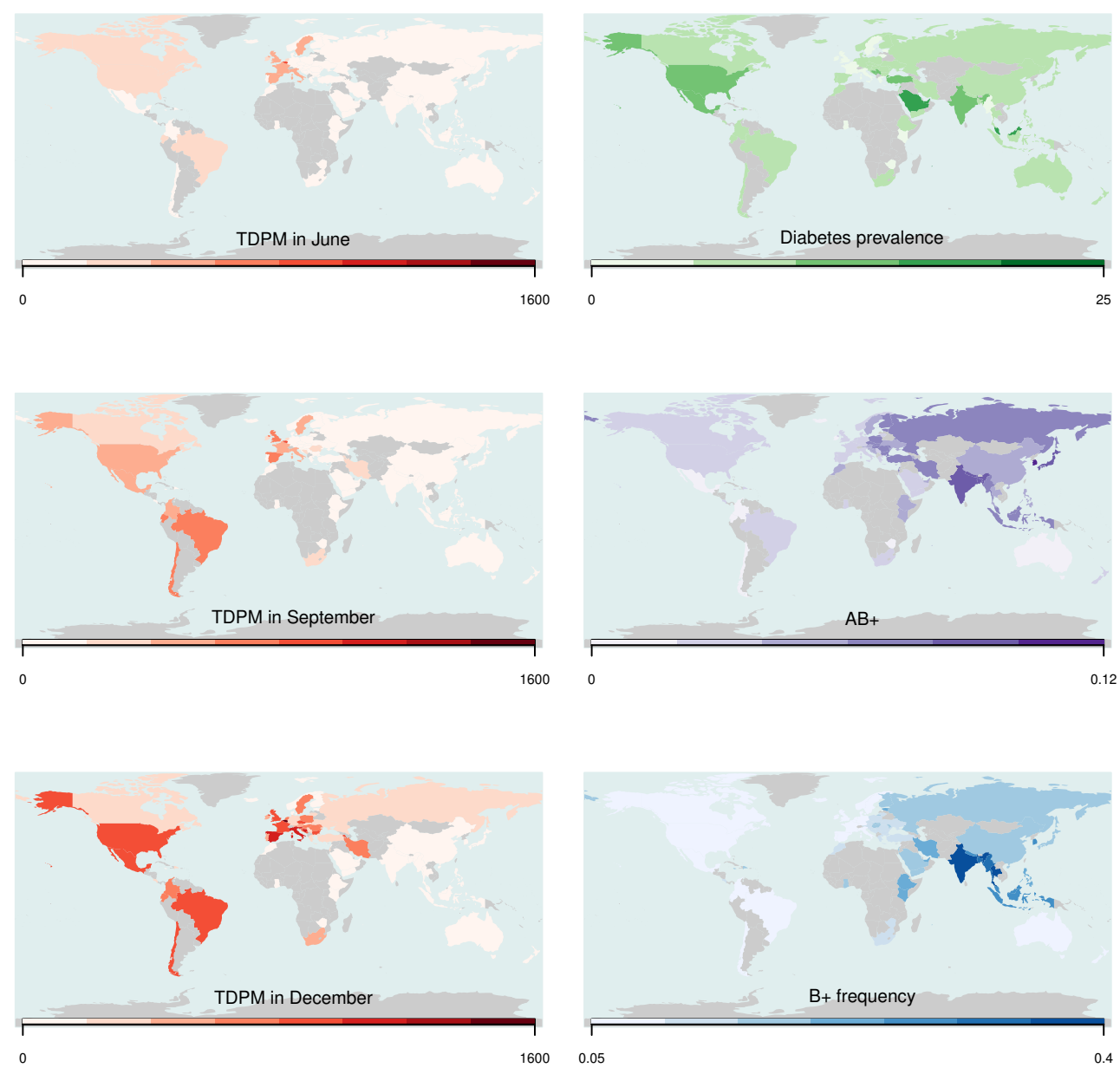

FIG. 3. Map of the TDPM in June, September, and December 2020 on the left. Maps of some of the input features on the right. Countries not included in the analysis are colored in gray.

caused by COVID-19 (TDPM), assessed in three different stages of the pandemic, a set of 12 selected country-level determinants and $\mathrm{ABO}$ and $\mathrm{Rh}$ blood group frequencies. Previous reports have analyzed the correlation of several variables with national case fatality rates but were limited to the first outbreak of COVID-19 (first half of 2020). To investigate putative predictors of TDPM, we used a non linear machine learning model combined with a feature selection procedure. Our model shows that the frequency of " $\mathrm{B}+$ " in the population is an important predictor of the TDPM in June, September, and December 2020. A multivariate linear model confirmed the significance of the " $\mathrm{B}+$ " frequency predictor as protective against death by COVID-19. The " $\mathrm{B}+$ " is protective because it is negatively correlated with the TDPM as displayed in Fig. 2. RF outperformed the linear model as it can be deduced comparing tables IV and V, which proves the existence of a complex (and not just linear) relationship between the input features and the outcome variable. Other factors emerged also as important to predict the TDPM although the model found them less important than frequency of "B+" and also their ability to predict the TDPM was not consistent over time: "Diabetes prevalence" and "Cardiovascular death rate" were important in June but not in September and December, in September frequency of "A-" switched from being tentative to being important, and stayed important in December together with "O-", the ratio "Rh/ Rh+", "Percentage of female smokers", and "Population density" . 
TABLE V. Performance metrics of a linear multivariate model applied to the set of features selected by Boruta at each time point, using all countries, averaged over 5 runs of cross validation (with the respective standard deviations). The last column reports only significant features. Significance codes: '***' 0.001, "**, 0.01 , and '*' 0.05 . The multivariate linear model found feature "B+" to be significant at all three time points, and also found "Cardiovascular death rate" to be significant but only in June. The significance of these features is higher in June and lower but similar in September and December, however most of the linearity is explained by the intercept of the linear model.

\begin{tabular}{|c|c|c|c|c|}
\hline Time point & $\mathbf{R}^{2}$ & RMSE & MAE & significant features \\
\hline June & $0.31 \pm 0.10^{* * *}$ & $138 \pm 49$ & $105 \pm 36$ & $\mathrm{~B}+{ }^{* *}$, Cardiovascular death rate $* *$ \\
September & $0.32 \pm 0.15^{* * *}$ & $184 \pm 36$ & $149 \pm 34$ & $\mathrm{~B}+{ }^{* *}$ \\
December & $0.29 \pm 0.17^{* * *}$ & $329 \pm 64$ & $260 \pm 44$ & $\mathrm{~B}+{ }^{*}$ \\
\hline
\end{tabular}
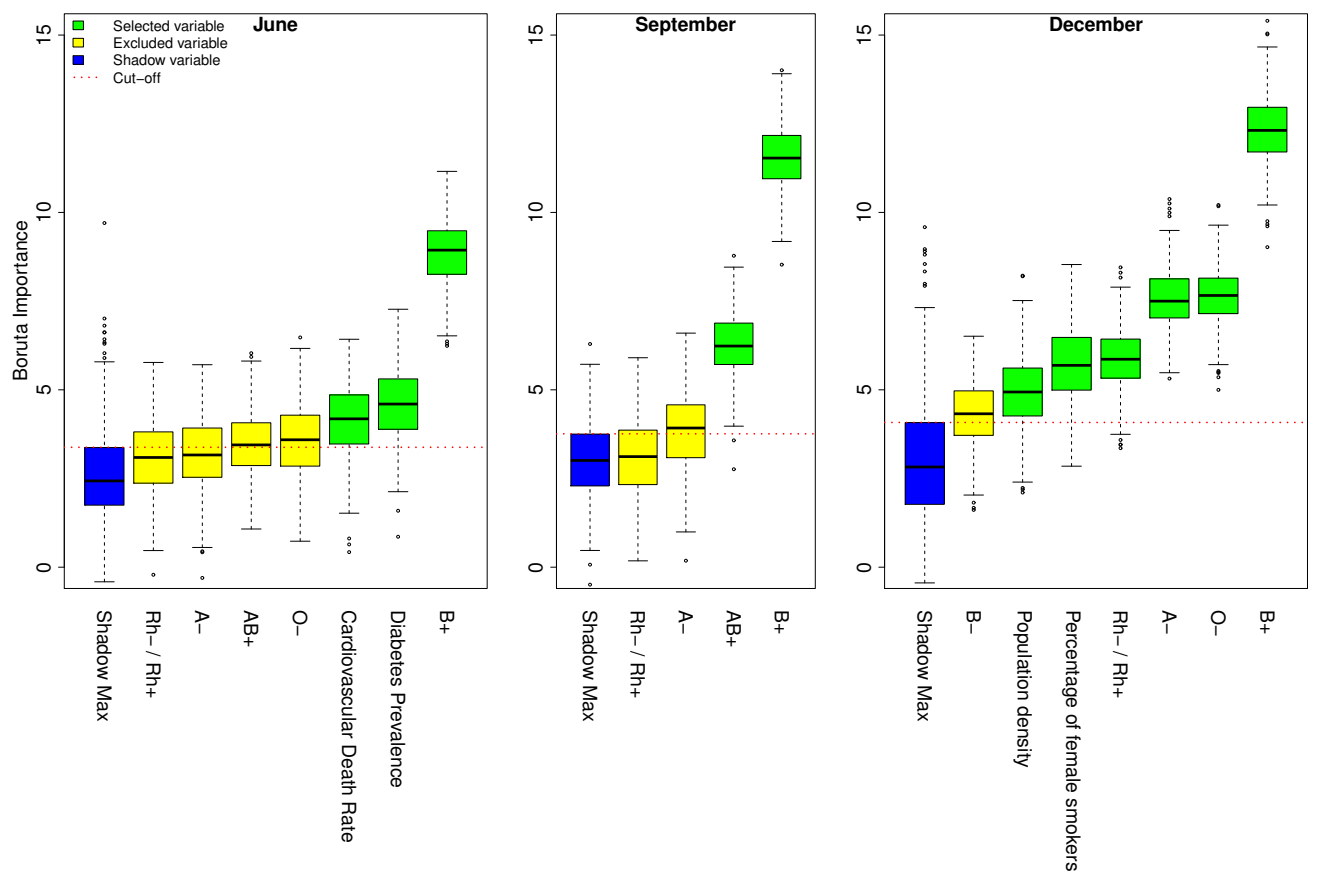

FIG. 4. Boxplot of the distribution of the Boruta importance measure for input variables with median higher than variable "Shadow Max". The distribution was obtained from 500 runs of the algorithm on the complete set of features using June, September, and December 2020 TDPM data. Using as cut-off the upper quartile of "Shadow Max", we colored in yellow excluded variables and in green variables selected for further analysis.

Interestingly, as the pandemic progressed, the number of important features predicted by Boruta grew to 6 in December (Fig. 4). Once more, the "B+" blood type frequency was the only feature always present, "A-" frequency was present twice, while no other feature had multiple occurrences. Thus, the putative role of $\mathrm{ABO}$ and $\mathrm{Rh}$ blood groups as determinants of countries TDPM seems to become more important with the progression of the pandemic.

The ABO gene locus encodes for a protein responsible for the different $\mathrm{ABO}$ blood types. In fact, functional $\mathrm{A}$ and $\mathrm{B}$ alleles at the $\mathrm{ABO}$ genetic locus express $\mathrm{A}$ or $\mathrm{B}$ transferases (AT and $\mathrm{BT}$ respectively) which are able to add a different glycosyl group to the $\mathrm{H}$ antigene. The $\mathrm{O}$ allele lacks this enzymatic activity due to a truncating mutation. Very recently, the ABO plasma protein levels have been associated with COVID-19 susceptibility and severity ${ }^{26}$. However, it is still unclear how the ABO protein modifies the COVID-19 risk. The intragenic rs505922 SNP has been shown to be responsible for differential ABO protein levels with an increasing effect for allele "C" and diminishing levels for allele " $\mathrm{T}$ "27,28. rs 505922 is in 

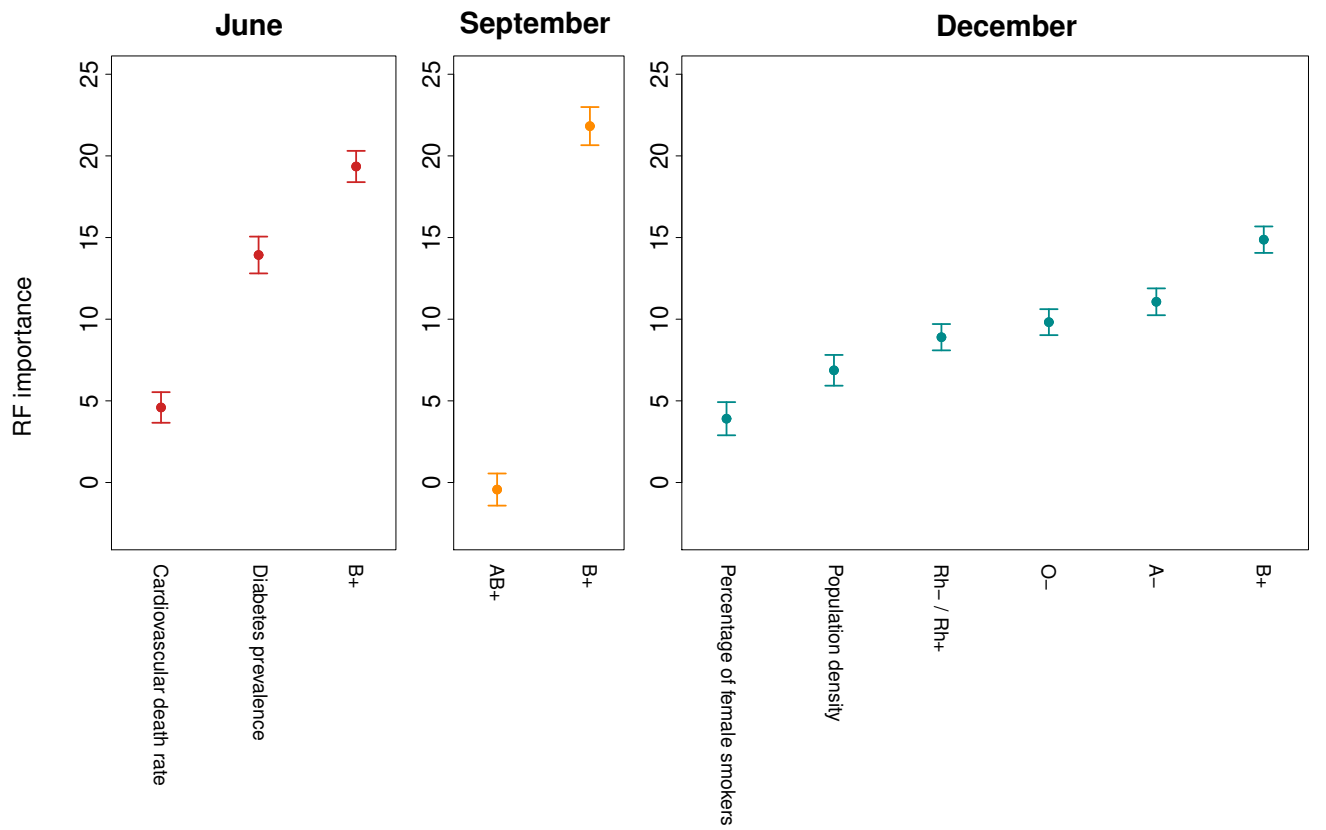

FIG. 5. Average importance of the variables used in the RF model over 100 runs of the RF algorithm, with the respective standard deviations.

LD with the O blood group SNP rs8176719, which has been repeatedly associated with an increased risk of venous thromboembolism ${ }^{29-32}$. The $r s 8176719$ polymorphism has been also associated to Factor VIII levels ${ }^{33}$, malaria ${ }^{34,35}$, venous thromboembolism ${ }^{36}$, vWF levels ${ }^{33}$. Similarly, recent findings suggest that the Rh blood group might be associated with severity of COVID-19, with "Rh-" having a protective role ${ }^{24,25}$. The increasing predominance of B+ as negatively associated to COVID-19 deaths, was paralleled by the disappearing of factors such as "diabetes prevalence" or "cardiovascular death rate". Thus, it might be possible that in the initial phase of the pandemic, severe COVID-19 was targeting categories with favoring comorbidities. In later waves of the pandemic, with increasing acquired immunization possibly protecting this group of subjects, individual genetics could have played a larger role in the fatal outcome of COVID-19. Further, recent work reported that both B and Rh+ were protective against influenza due to identified zoonotic or pandemic influenza virus ${ }^{37}$. $\mathrm{B}$ was also protective against pneumonia due to S. pneumoniae. Finally, the B blood group has a decreasing gradient of frequency from East Asia going westward. Thus, it is possible that the B allele has been under positive selection pressure by an ancient viral epidemic, which shaped the ancestral eastern Asian genome ${ }^{38}$.

This study has several limitations. We limited our analyses to determinants included in the OWID series, hence it is possible that additional factors might explain the TDPM differences observed between countries. For example, vaccination against Bacillus CalmetteGuerin (BCG) has been recently reported as protective against severe COVID-19 infection ${ }^{39-42}$. However, a recent work has reported that, similarly with several determinants we investigated, BCG vaccination exerts a strong protective effect against COVID-19 in the early stage of the pandemic while fading in later stages ${ }^{43}$. Finally, we investigated only the ABO and Rhesus blood groups amongst many genetic loci that have been recently identified in genome wide association studies (GWAS) ${ }^{14,44}$, or more focused approaches ${ }^{11,12,14,21}$. However, $\mathrm{ABO}$ and $\mathrm{Rh}$ are part of a very limited group of genetic loci for which frequencies of the different phenotypic classes are available for almost all countries in the world. For the vast majority of SNPs only ethnic-specific frequencies can be extracted from available databases. In conclusion, differently from previous studies, in our investigation, the in- 
fluence of genetic and non-genetic factors on the TDPM has been evaluated in different stages of the COVID-19 pandemic. Our findings suggest that in more advanced stages of the pandemic, individual genetic factors might exert a stronger influence on COVID-19 severity.

\section{DATA AVAILABILITY}

All relevant data are available from the authors with reasonable requests.

\section{REFERENCES}

${ }^{1}$ WHO cov-19 interactive timeline. https://www.who.int/emergencies/diseases/ novel-coronavirus-2019/interactive-timeline. Accessed: 2021-04-19.

${ }^{2}$ JHU cov-19 map. https://coronavirus.jhu.edu/map.html. Accessed: 2021-04-19.

${ }^{3}$ OWID data on the coronavirus pandemic. https://ourworldindata.org/mortality-risk-covid.

${ }^{4}$ Flint, S. \& Tahrani, A. Covid-19 and obesity-lack of clarity, guidance, and implications for care. Lancet Diabetes Endocrinology 8, 474-475 (2020).

${ }^{5}$ Zhou, Y., Chi, J., Lv, W. \& Wang, Y. Obesity and diabetes as high-risk factors for severe coronavirus disease 2019 (covid-19). Diabetes Metab. Res. Rev. 37, e3377 (2021).

${ }^{6}$ Richardson, S. \& et al. Presenting characteristics, comorbidities, and outcomes among 5700 patients hospitalized with covid-19 in the new york city area. JAMA 323, 2052-2059 (2020).

${ }^{7}$ Zaki, N., Alashwal, H. \& Ibrahim, S. Association of hypertension, diabetes, stroke, cancer, kidney disease, and high-cholesterol with covid-19 disease severity and fatality: A systematic review. Diabetes Metab. Syndr. 14, 1133-1142 (2020).

${ }^{8} \mathrm{Ji}$, Y., Ma, Z., Peppelenbosch, M. P. \& Pan, Q. Potential association between covid-19 mortality and health-care resource availability. Lancet Glob. Health 8, e480 (2020).

${ }^{9}$ Kenyon, C. Flattening-the-curve associated with reduced covid-19 case fatality rates- an ecological analysis of 65 countries. J. Infect. 81, e98-e99 (2020).

${ }^{10}$ Dowd, J. B. \& et al. Demographic science aids in understanding the spread and fatality rates of covid-19. PNAS 117, 9696-9698 (2020).

${ }^{11} \mathrm{Hou}$, Y. J. \& et al. Sars-cov-2 reverse genetics reveals a variable infection gradient in the respiratory tract. Cell 182, 429-446.e14 (2020).

${ }^{12}$ Zhang, X. \& et al. Viral and host factors related to the clinical outcome of covid-19. Nature 583, 437-440 (2020).

${ }^{13}$ Di Maria, E., Latini, A., Borgiani, P. \& Novelli, G. Genetic variants of the human host influencing the coronavirus-associated phenotypes (sars, mers and covid-19): rapid systematic review and field synopsis. Human Genomics 14, 1-19 (2020).

${ }^{14}$ Zeberg, H. \& Pääbo, S. The major genetic risk factor for severe covid-19 is inherited from neanderthals. Nature 610-612 (2020).

${ }^{15}$ Blood type distribution by country. Retrieved from https://en.wikipedia.org/wiki/Blood_type_ distribution_by_country on 2021-01-16.

${ }^{16}$ The complete our world in data covid-19 dataset. Retrieved from https://github.com/owid/ covid-19-data/tree/master/public/data on 2021-01-16.

${ }^{17}$ Kursa, M. B. \& Rudnicki, W. R. Feature selection with the boruta package. Journal of Statistical Software 36, 1-13 (2010).

${ }^{18}$ Breiman, L. Random forests. Machine Learning 45, 32-45 (2001).

${ }^{19} \mathrm{R}$ Core Team. R: A Language and Environment for Statistical Computing. R Foundation for Statistical Computing, Vienna, Austria (2020). URL https://www.R-project.org/.

${ }^{20}$ Ellinghaus, D. \& et al. Genome wide association study of severe covid-19 with respiratory failure. $N$ Engl J Med 283, 1522-34 (2020).

${ }^{21}$ Shelton, J. F. \& et al. Trans-ethnic analysis reveals genetic and non-genetic associations with covid-19 susceptibility and severity. medRxiv (2020).

${ }^{22}$ Zhao, J. \& et al. Relationship between the abo blood group and the covid-19 susceptibility. Clin. Infect. Dis. ciaa1150 (2020).

${ }^{23} \mathrm{Wu}$, Y., Feng, Z., P., L. \& Yu, Q. Relationship between the abo blood group and the covid-19 susceptibility. Clin. Chim. Acta 509, 220-3 (2020).

${ }^{24}$ Ray, J. G., Schull, M. J., Vermeulen, M. J. \& Park, A. L. Association between abo and rh blood groups and sars-cov-2 infection or severe covid-19 illness: A population-based cohort study. Ann. Intern. Med. 174, 308-315 (2021).

${ }^{25}$ Zietz, M., Zucker, J. \& Tatonetti, N. P. Associations between blood type and covid-19 infection, intubation, and death. Nat. Commun. 11, 5761 (2020). 
${ }^{26}$ Hernandéz Cordero, A. I. e. a. Multi-omics highlights abo plasma protein as a causal risk factor for covid-19. Hum. Genet. 140, 969-979 (2021).

${ }^{27}$ Trégouët, D. A. \& et al. Common susceptibility alleles are unlikely to contribute as strongly as the $\mathrm{fv}$ and abo loci to vte risk: results from a gwas approach. Blood 113, 5298-303 (2009).

${ }^{28}$ Paré, G. \& et al. Novel association of abo histo-blood group antigen with soluble icam-1: results of a genome wide association study of 6,578 women. PLoS Genet 4, e1000118 (2008).

${ }^{29}$ Frischmuth, T. \& et al. Joint effect of multiple prothrombotic genotypes and obesity on the risk of incident venous thromboembolism. Thromb Haemost. (2021).

${ }^{30}$ Skille, H. \& et al. Combined effects of five prothrombotic genotypes and cancer on the risk of a first venous thromboembolic event. J. Thromb Haemost. 18, 2861-2869 (2020).

${ }^{31}$ Sejrup, J. K. \& et al. Myocardial infarction, prothrombotic genotypes, and venous thrombosis risk: The troms $\varnothing$ study. Res. Pract. Thromb Haemost. 4, 247-254 (2020).

${ }^{32}$ Groot, H. E. \& et al. Genetically determined abo blood group and its associations with health and disease. Arterioscler. Thromb Vasc. Biol. 40, 830-838 (2020).

${ }^{33}$ Sabater-Lleal, M. e. a. Genome-wide association transethnic meta-analyses identifies novel associations regulating coagulation factor viii and von willebrand factor plasma levels. Circulation 139, 620-635 (2019).

${ }^{34}$ Timmann, C. \& et al. Genome-wide association study indicates two novel resistance loci for severe malaria. Nature 489, 443-446 (2012).

${ }^{35}$ Band, G. \& et al. Malaria genomic epidemiology network. insights into malaria susceptibility using genome-wide data on 17,000 individuals from africa, asia and oceania. Nat. Comm. 10, 5732 (2019).

${ }^{36} \mathrm{Heit}$, J. A. \& et al. A genome-wide association study of venous thromboembolism identifies risk variants in chromosomes 1q24.2 and 9q. Journal of Thrombosis and Haemostasis: JTH 10, 1521-1531 (2012).

${ }^{37}$ Dahlén, T., M., C., Zhao, J., Olsson, M. L. \& Edgren, G. An agnostic study of associations between abo and rhd blood group and phenome-wide disease risk. Elife 10, e65658 (2021).

${ }^{38}$ Souilmi, Y. \& et al. An ancient viral epidemic involving host coronavirus interacting genes more than 20,000 years ago in east asia. bioRxiv (2021).

${ }^{39}$ Patella, V., Delfino, G., Bruzzese, D., Giuliano, A. \& Sanduzzi, A. The bacillus calmette-guérin vaccination allows the innate immune system to provide protection from severe covid-19 infection. PNAS 117, $25205-25206$ (2020).

${ }^{40}$ Escobar, L. E., Molina-Cruz, A. \& Barillas-Mury, C. Bcg vaccine protection from severe coronavirus disease 2019 (covid-19). PNAS 117, 17720-17726 (2020).

${ }^{41}$ Klinger, D., Blass, I., Rappoport, N. \& Linial, M. Significantly improved covid-19 outcomes in countries with higher bcg vaccination coverage: A multivariable analysis. Vaccines 8, 523 (2020).

${ }^{42}$ Brooks, N. A. e. a. The association of coronavirus disease-19 mortality and prior bacille calmette-guerin vaccination: A robust ecological analysis using unsupervised machine learning. Sci. Rep. 11, 1-9 (2021).

${ }^{43} \mathrm{Fu}, \mathrm{W}$. \& et al. Reconcile the debate over protective effects of bcg vaccine against covid-19. Sci. Rep. 11, 8356 (2021).

${ }^{44}$ Pairo-Castineira, E. \& et al. Genetic mechanisms of critical illness in covid-19. Nature 591, 92-98 (2021).

${ }^{45}$ Guan, W. \& et al. Comorbidity and its impact on 1590 patients with covid-19 in china: a nationwide analysis. European Respiratory Journal 55 (2020).

\section{AUTHOR CONTRIBUTIONS}

Conceptualization, A.M., E.P. and A.S.; methodology, A.M., E.P. and A.S.; software, A.M. and E.P.; formal analysis, A.M. and E.P.; writing - original draft preparation, A.M., E.P. and A.S.; writing - review and editing, N.A., L.B., R.B., A.M., E.P., and A.S.; visualization, A.M. and E.P. All authors have read and agreed to the published version of the manuscript. 
Figures

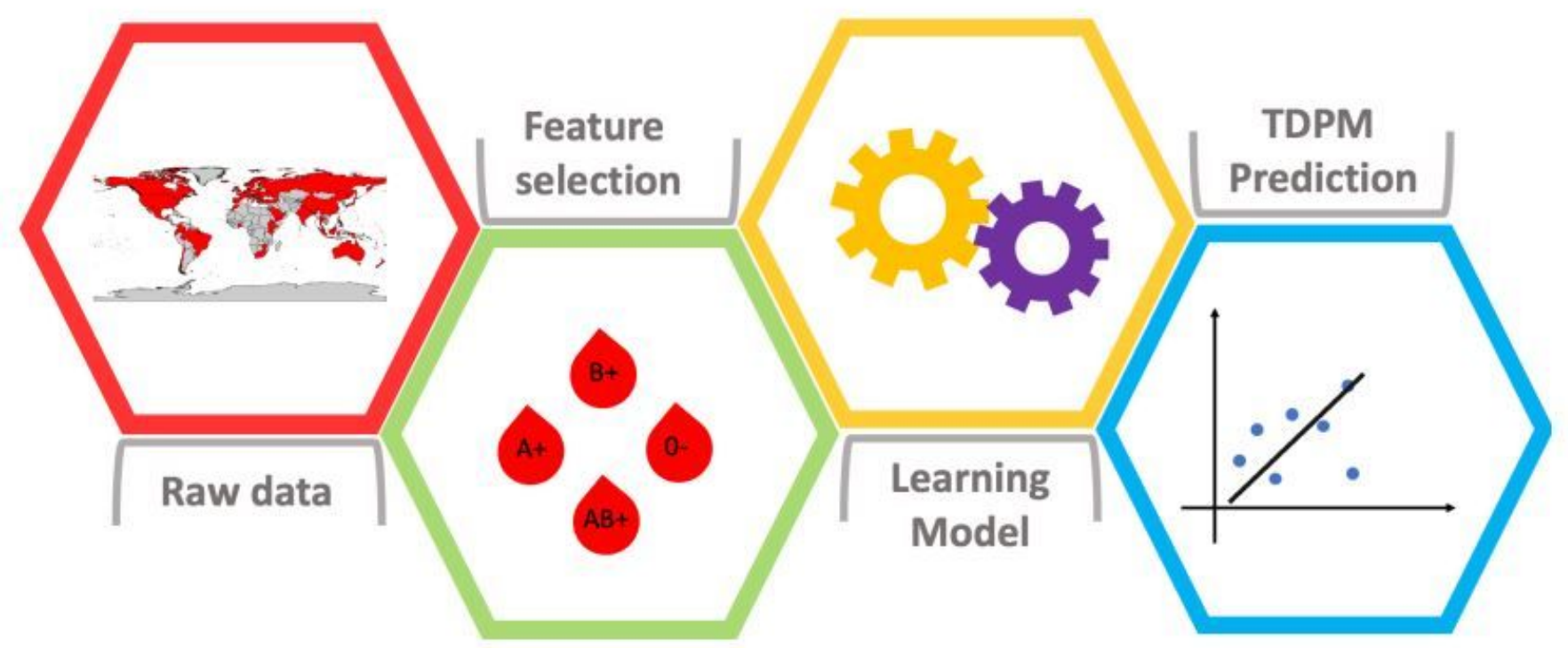

Figure 1

Flowchart of the proposed methodology. We fed the learning algorithm with selected features to forecast the TDPM. 


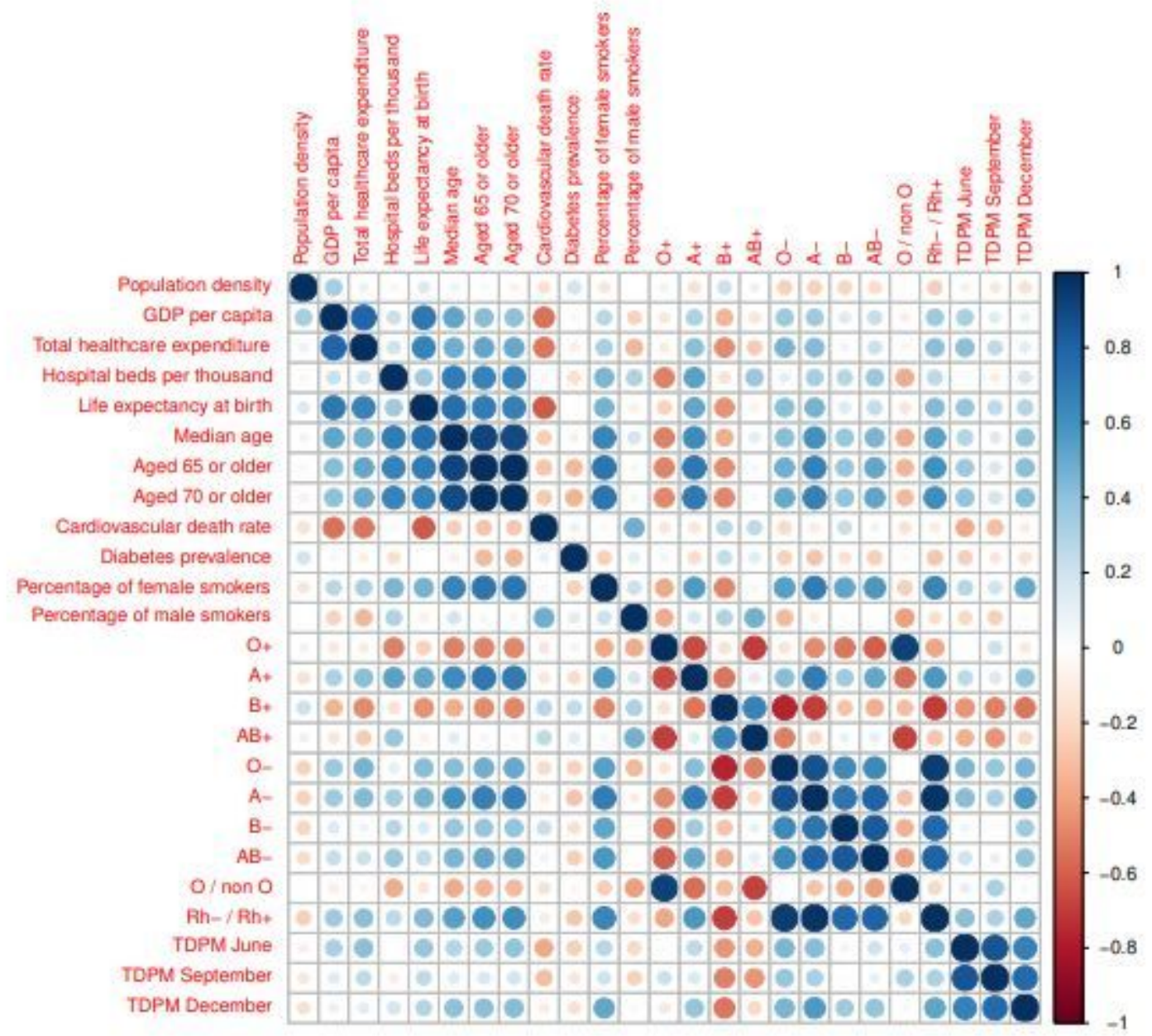

Figure 2

Correlation matrix of all variables (both dependent and independent). As expected "Median age of the population", "Population aged 65 or older", and "Population aged 70 or older" have mutual correlation close to 1; also the genetic features are highly correlated with each other. The TDPMs at the three different dates are also highly correlated with each other, as expected. Notably, the "B+" predictor has the highest (and negative) linear correlation with the TDPM at all three dates. 

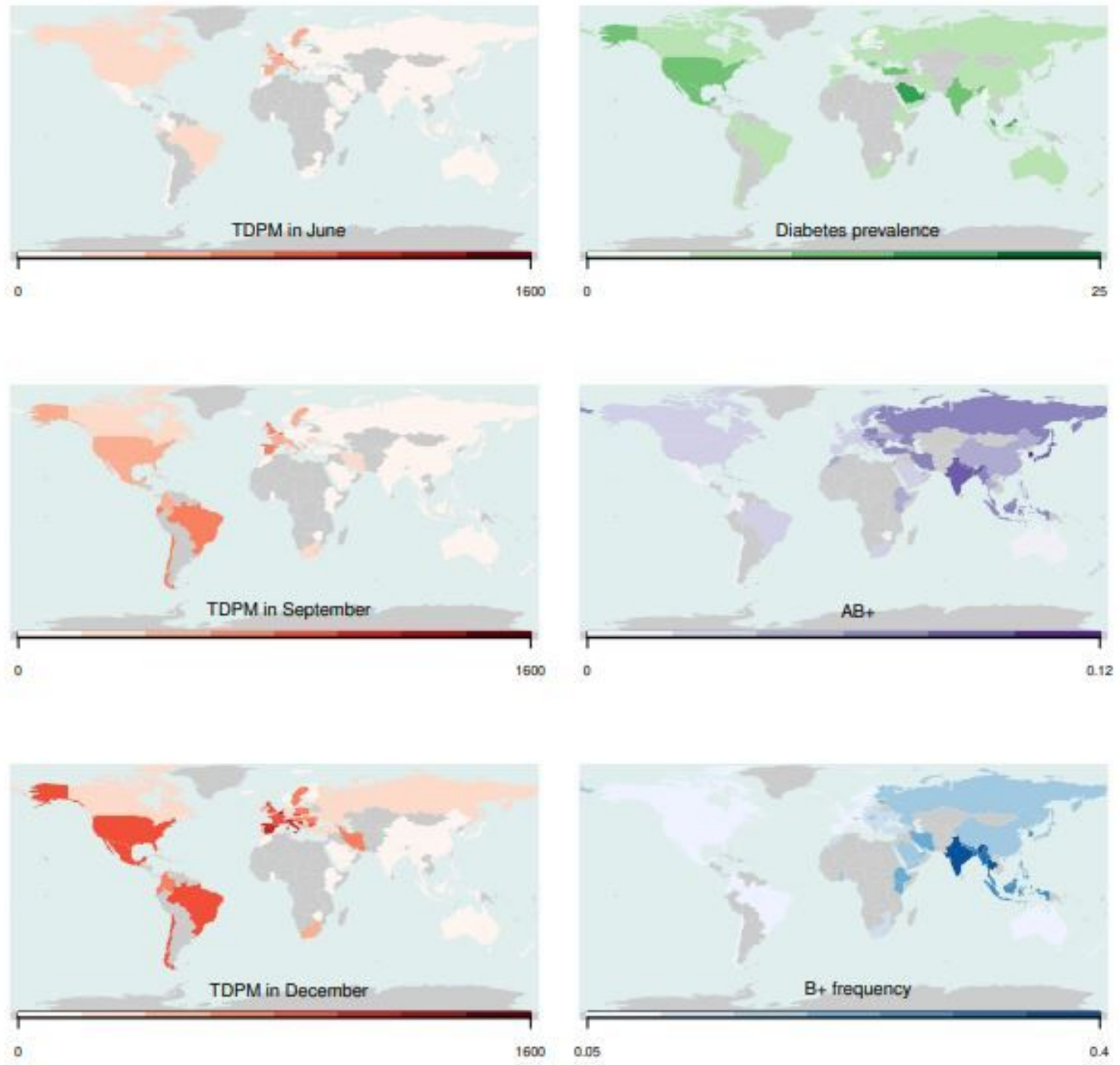

\section{Figure 3}

Map of the TDPM in June, September, and December 2020 on the left. Maps of some of the input features on the right. Countries not included in the analysis are colored in gray Note: The designations employed and the presentation of the material on this map do not imply the expression of any opinion whatsoever on the part of Research Square concerning the legal status of any country, territory, city or area or of its authorities, or concerning the delimitation of its frontiers or boundaries. This map has been provided by the authors. 

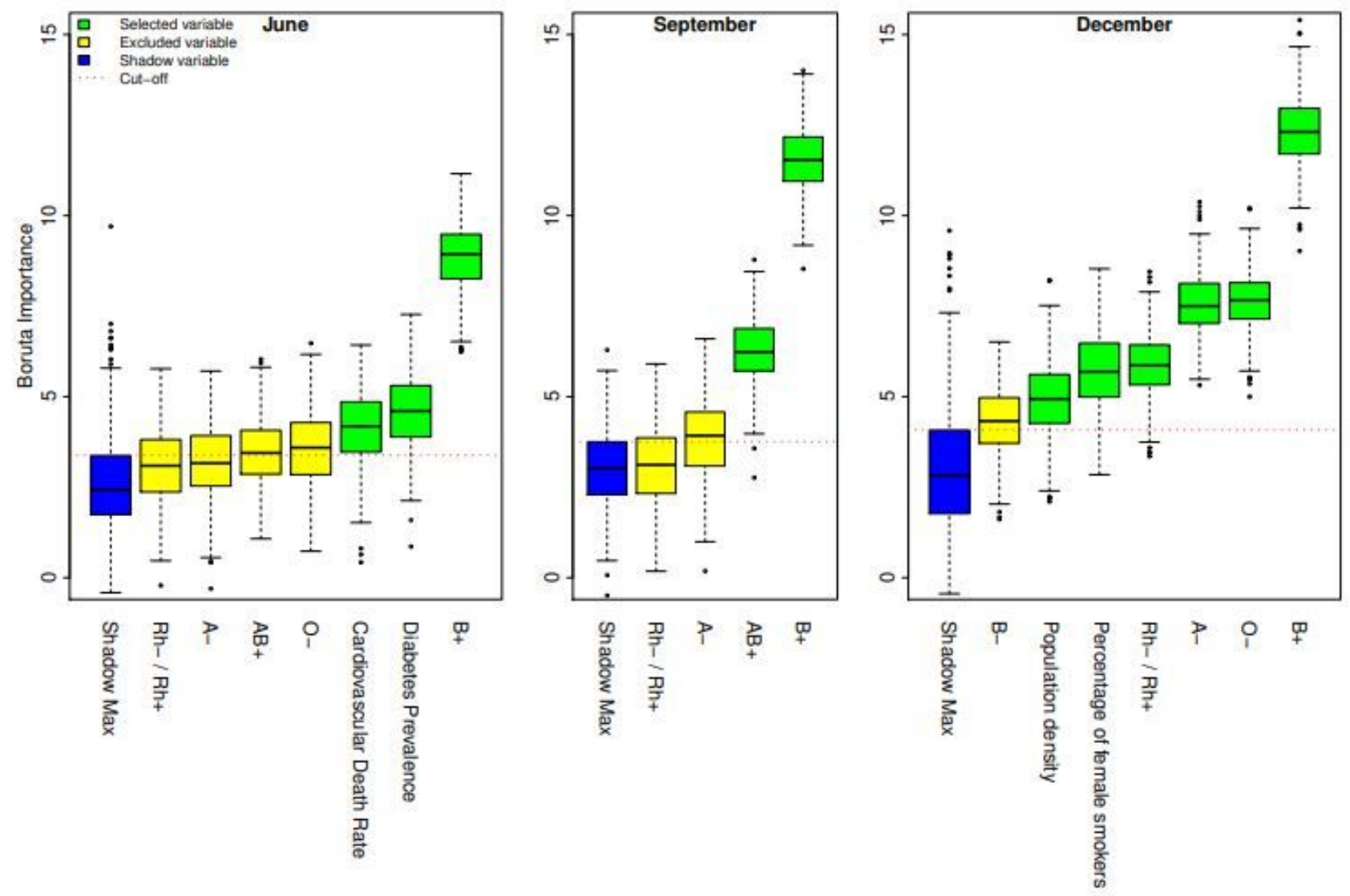

Figure 4

Boxplot of the distribution of the Boruta importance measure for input variables with median higher than variable "Shadow Max". The distribution was obtained from 500 runs of the algorithm on the complete set of features using June, September, and December 2020 TDPM data. Using as cut-off the upper quartile of "Shadow Max", we colored in yellow excluded variables and in green variables selected for further analysis. 


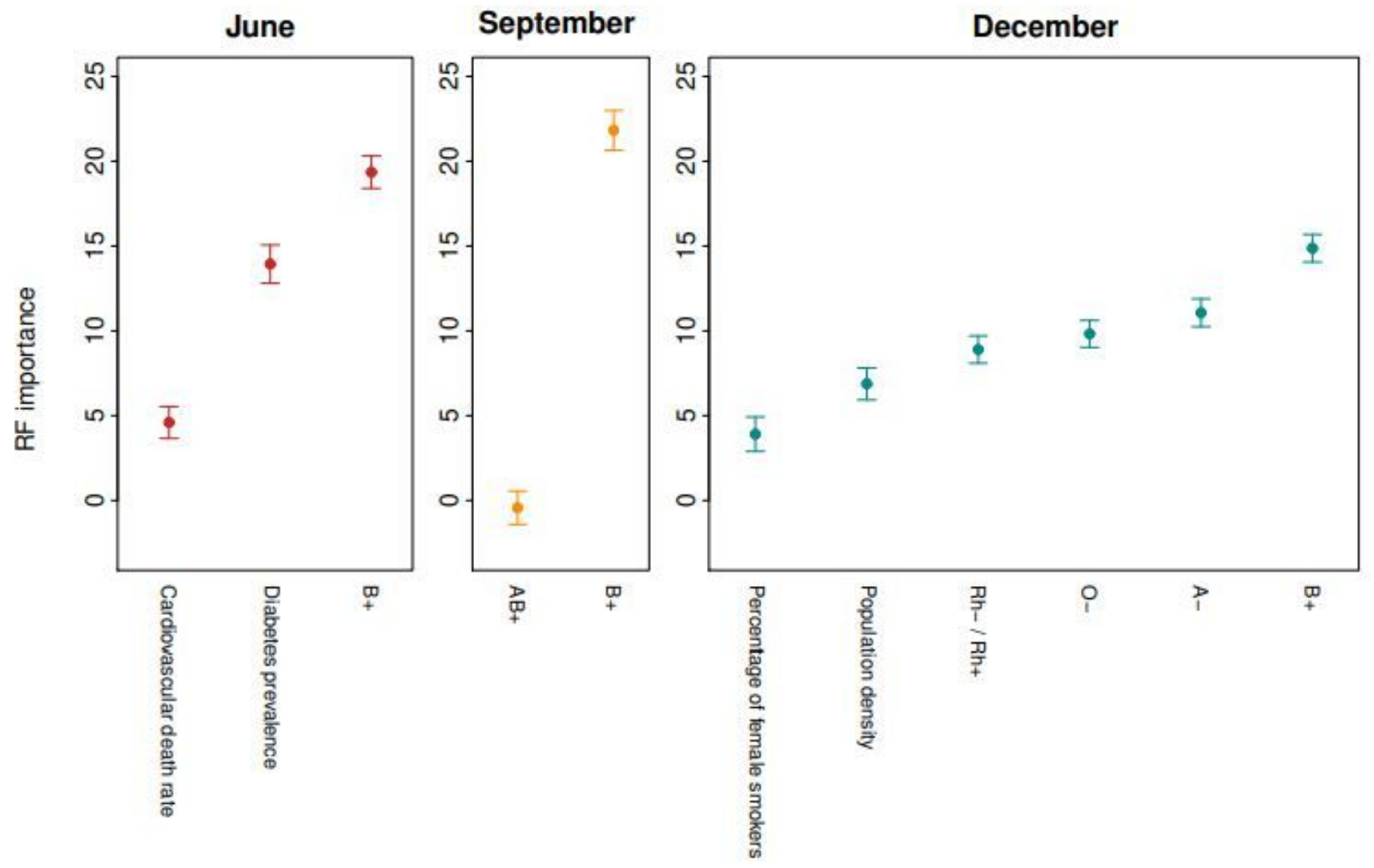

Figure 5

Average importance of the variables used in the RF model over 100 runs of the RF algorithm, with the respective standard deviations.

\section{Supplementary Files}

This is a list of supplementary files associated with this preprint. Click to download.

- SupplementaryInformationtoCountrylevel....pdf 